\title{
Postinfectious Inflammatory Syndrome following Cryptosporidium Infection
}

\author{
Matthew Agnewa Anita Kottapallia Ven Kottapallib \\ aUniversity of Toledo College of Medicine, Toledo, $\mathrm{OH}$, USA; ${ }^{b} \mathrm{GI}$ Physicians Inc., Lima, $\mathrm{OH}$, \\ USA
}

Keywords

Cryptosporidium postinfectious inflammatory syndrome

\begin{abstract}
A 38-year-old woman with a history of Crohn's disease, multiple bowel resections, and ileostomy placement presented to the hospital with symptoms of increased ileostomy output for 1 week. She reported that she was emptying her bag fifteen times a day as opposed to her normal 3-4 times a day. Upon workup, she was found to have an acute kidney injury (AKI), and stool studies were positive for Cryptosporidium. She was treated with nitazoxanide 500 $\mathrm{mg}$ p.o. BID for 3 days along with continued rehydration. The patient was discharged after creatinine $(\mathrm{Cr})$ and electrolytes returned to baseline. She continued to have elevated ileostomy output, and 1 week later, she was readmitted for another AKI and worsening of symptoms. At this hospitalization, stool studies were negative for Cryptosporidium, and the gastroenterologist consult recommended evaluation for active Crohn's and Lomotil for possible short bowel syndrome. Eventually, her laboratory results improved, and she was discharged again before the full workup was completed. The patient's ileostomy output continued to remain high following the second hospital discharge, and she eventually returned with another AKI, her third visit in a month. The workup for active Crohn's was completed, with fecal calprotectin, serum cortisol, and small bowel follow-through all returning to normal. At this time, postinfectious inflammatory syndrome was suspected, and she was started on $60 \mathrm{mg}$ of prednisone for 2 weeks. Steroid therapy elicited a significant response with normalization of her ileostomy output and return of laboratory results to baseline. The patient was discharged without return of symptoms at follow-up.
\end{abstract}

\section{Karger $\stackrel{N}{\%}$}


Agnew et al.: Postinfectious Inflammatory Syndrome following Cryptosporidium Infection

\section{Introduction}

The following case illustrates the course of acute kidney injuries (AKIs) in a 38-year-old female patient with Crohn's disease and a history of multiple bowel resections due to her disease. Crohn's disease is an inflammatory bowel disease characterized by transmural inflammation that occurs in a noncontiguous fashion along the gastrointestinal tract. Treatment of these patients may involve medical management targeting the associated inflammatory process, while many patients also require surgical resection of the affected bowel.

In patients with these gastrointestinal comorbidities, the workup involves multiple considerations. Stool studies for investigation of an infectious etiology of illness should be ordered. Short bowel syndrome should be considered with a history of bowel resection, due to patients having a lack of functional bowel and associated malabsorption sequelae. Additionally, active Crohn's disease must be excluded. In complicated clinical courses, as common pathologies are ruled out, less common entities should be considered. Postinfectious inflammatory syndrome is one such uncommon scenario. This syndrome is observed in patients who fail to improve following recovery from a known infection. Postinfectious inflammatory syndrome involves an aggravated immune response and ongoing inflammation following resolution of an infection. This condition can exist across organ systems. In the gastrointestinal system, this inflammation following infections can present as irritable bowel-like symptoms, diarrhea, constipation, fever, and/or vomiting, among others [1].

\section{Case Report/Case Presentation}

The case patient is a 38-year-old Caucasian woman with a medical history of Crohn's disease (diagnosed 2003) and a surgical history of right colon resection with distal ileal resection and ileocolonic anastomosis (2010), 16 inches of jejunal resection (2010), followed by ileostomy placement (2016) complicated by diversion colitis, and treated with medium-chain fatty acid enemas. The patient, previously on human biologics including Humira and Remicade, was on Entyvio $300 \mathrm{mg}$ IV every 2 months at the time of case presentation.

She first presented to the hospital in the fall of 2019 with complaints of increased ileostomy output for approximately 1 week. The patient also complained of feelings of weakness, fatigue, dehydration, and epigastric pain. She reported experiencing severe ileostomy output, emptying her bag 10-15 times a day compared to her normal 3 or 4 times a day (at 500-750 mL for each change) in the week, leading to her hospital admission.

Upon workup, she was found to have an AKI with a creatinine (Cr) 2.7 (baseline $\mathrm{Cr}$ of 1.1 3 months prior), BUN of 21, and stool studies positive for Cryptosporidium. The gastroenterologist (GI) consult recommended nitazoxanide $500 \mathrm{mg}$ p.o. BID for 3 days along with continued rehydration (IV fluids, clear fluids, and Pedialyte 3 times daily) and octreotide 50 mcg subcutaneously q.12 h to reduce bowel motility due to possible comorbid short bowel syndrome. Additionally, a small bowel follow-through to rule out fistula formation due to possible Crohn's exacerbation was recommended. The small bowel follow-through, however, was not performed nor was octreotide given due to an issue with precertification and insurance. The patient was discharged 2 days following admission after her $\mathrm{Cr}$ and electrolytes returned to their reference ranges.

Following discharge, the patient continued to have increased ileostomy output along with severe nausea and vomiting with immediate onset upon eating. Approximately 1 week following her discharge, her $\mathrm{Cr}$ was elevated to 6.9 for which she was admitted to the

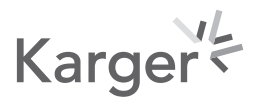


Agnew et al.: Postinfectious Inflammatory Syndrome following Cryptosporidium Infection

Fig. 1. Figures are from the small-bowel follow-through that was performed during the patient's third hospital admission to evaluate for active Crohn's disease. There were no signs of new strictures or fistula formation. Additionally, the imaging was consistent with her previous imaging studies and past abdominal surgeries. Based upon this imaging and the other laboratory studies, active Crohn's disease was ruled out.

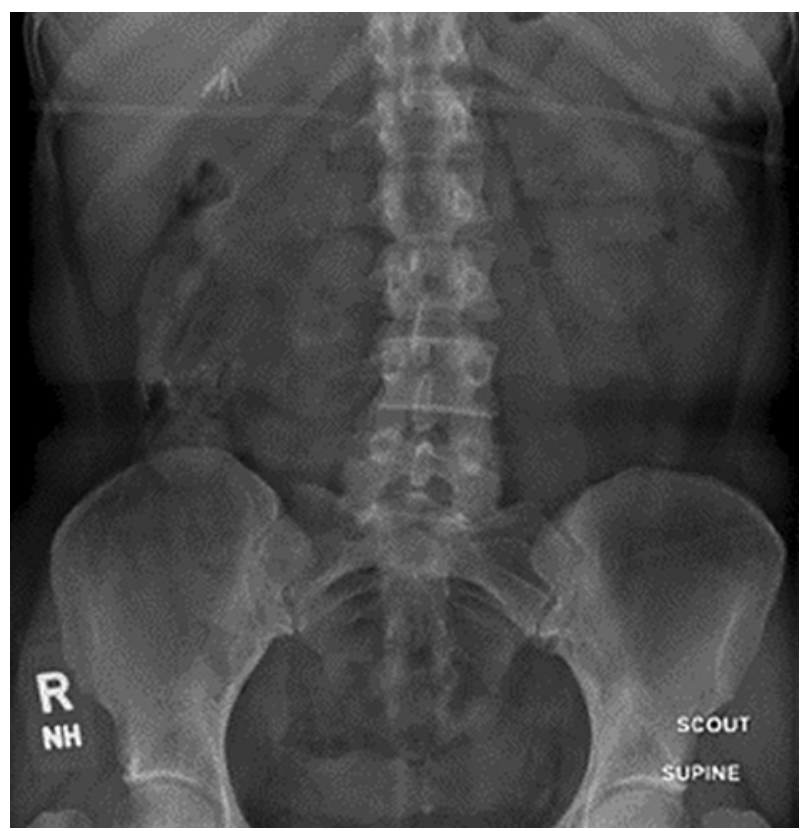

hospital by her primary care team. Initial laboratory studies at this second admission showed Cr 8.6, Na 122, K 3.6, Cl 81, CO2 16, and BUN 61. Stool studies for recurrent cryptosporidium were negative, and she was again treated for AKI via rehydration and electrolyte replacement. Although her laboratory values continued to improve with fluid bolus, her ostomy output was approximately $400 \mathrm{~mL}$ every $2 \mathrm{~h}$ and as high as 3,000 $\mathrm{mL}$ of green fluid in 1 day. The patient was also given Lomotil to slow the bowels, and the GI consult at this time recommended subcutaneous octreotide along with small bowel follow-through, fecal calprotectin, and serum cortisol levels for evaluation of active Crohn's disease versus short bowel syndrome. Octreotide again failed approval by insurance, and small bowel followthrough was not completed prior to the patient's discharge upon her laboratory results normalizing over the course of 6 days.

Following the second discharge, ostomy output remained high with the patient emptying her bag 8 times a day with approximately $200 \mathrm{~mL}$ of fluid each time. Her previous symptoms remained, and follow-up blood work less than a week later showed a $\mathrm{Cr}$ of 7.9, BUN of 65, and decreased electrolytes. This resulted in the third hospital admission of AKI within the month. Nephrology at this time was considering putting the patient on dialysis. The GI consult at this admission resulted in a small bowel follow-through and approval of octreotide subcutaneous injections. Small bowel follow-through at this time was negative for active Crohn's, stricture, or fistula, as shown in Figures 1-3.

Calprotectin and cortisol levels were within their reference range. Postinfectious inflammatory syndrome was suspected at this time to be causing her AKIs, with elevated C-reactive protein supporting the diagnosis. The patient was subsequently started on $60 \mathrm{mg}$ of prednisone by mouth once daily for 2 weeks. Steroid therapy elicited a significant response in both Cr clearance and glomerular filtration rate which went from 10 to 60 and 5 to 64 , respectively. The patient was discharged after a week and tapered to $10 \mathrm{mg}$ of prednisone daily along with tincture opium $0.6 \mathrm{~mL} 3$ times daily (due to octreotide not being approved for outpatient treatment) for treatment of short bowel syndrome. She has been stable for 5 months since this episode and reports her ileostomy bag output to be within normal limits with formed stool appearing in the bag and a most recent $\mathrm{Cr}$ of 1.1 .

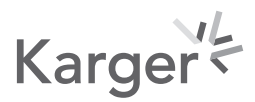


Agnew et al.: Postinfectious Inflammatory Syndrome following Cryptosporidium Infection

Fig. 2. Figures are from the small bowel follow-through that was performed during the patient's third hospital admission to evaluate for active Crohn's disease. There were no signs of new strictures or fistula formation. Additionally, the imaging was consistent with her previous imaging studies and past abdominal surgeries. Based upon this imaging and the other laboratory studies, active Crohn's disease was ruled out.

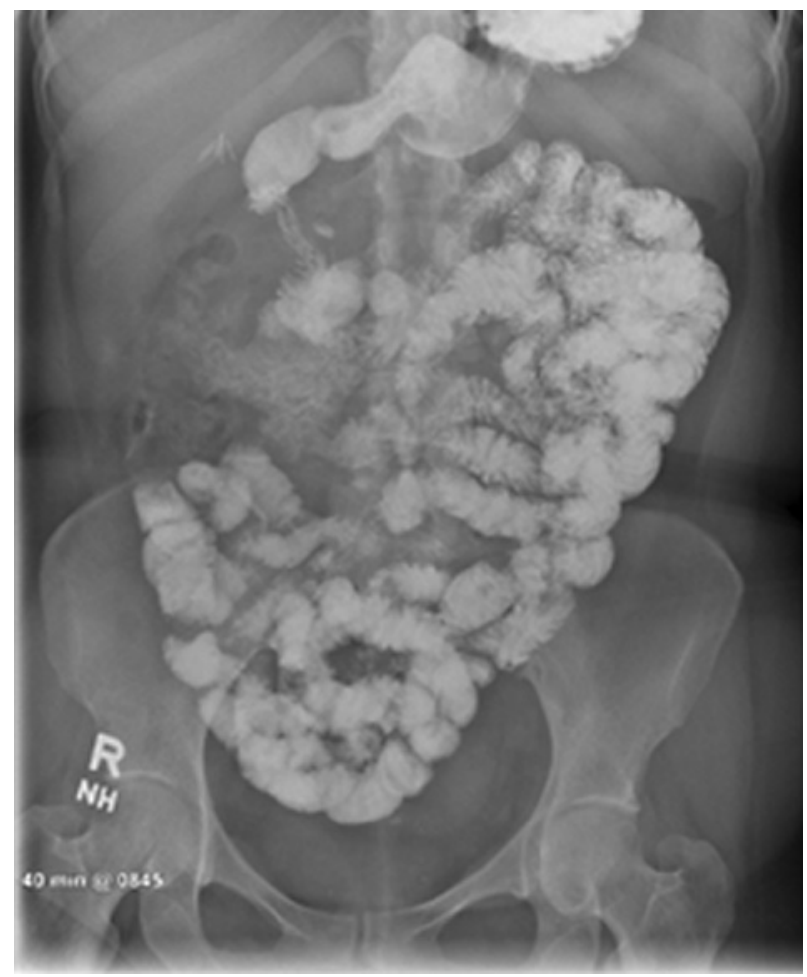

\section{Discussion/Conclusion}

This patient presentation illustrates the role of postinfectious inflammatory syndrome in the setting of AKI in a patient with Crohn's disease and a history of multiple GI comorbidities. Although little data exist on cryptosporidiosis in IBD patients, some evidence suggests the prevalence of cryptosporidium is greater among IBD patients than that previously assumed. One study in India showed 2\% of ulcerative colitis patients were positive for Cryptosporidium [2]. Additionally, mouse models have demonstrated the possibility of cryptosporidium infections to worsen or trigger an IBD flare-up [3, 4]. Marcial and Madara [5] in 1986 observed Cryptosporidium within the cytoplasm of M-cell enterocytes overlying Peyer's patches. It was initially posited that this location suggests an ability to activate the immune system in a way which IBD patients may be particularly susceptible. The onset of severe AKIs following initial infection would suggest that Cryptosporidium played a key role in this particular case. Although this patient was on immunosuppressive therapy, it is unlikely that this played a role in her severe AKIs. This is reflected in a study done during the 1993 Milwaukee outbreak of Cryptosporidium, during which twelve cases of Cryptosporidium presented in IBD patients with stable UC or CD. All cases recovered completely, with immunosuppressive therapy having no association with more severe illness [6].

While comorbidities such as Crohn's disease and short bowel syndrome are observed in this patient, we believe postinfectious inflammatory syndrome with renal involvement to be the primary pathology responsible for the clinical course described. The observed calprotectin, cortisol levels, and small bowel follow-through allowed exclusion of active Crohn's. Short bowel syndrome was addressed with loperamide prior to octreotide approval, although there is some evidence that high-output ileostomies may require more than IV fluids and standard doses of loperamide [7, 8]. While most clinical trials of loperamide do not exceed $16 \mathrm{mg} /$ day, a series of case reports in 2015 demonstrated doses of 
Agnew et al.: Postinfectious Inflammatory Syndrome following Cryptosporidium Infection

Fig. 3. Figures are from the small bowel follow-through that was performed during the patient's third hospital admission to evaluate for active Crohn's disease. There were no signs of new strictures or fistula formation. Additionally, the imaging was consistent with her previous imaging studies and past abdominal surgeries. Based upon this imaging and the other laboratory studies, active Crohn's disease was ruled out.

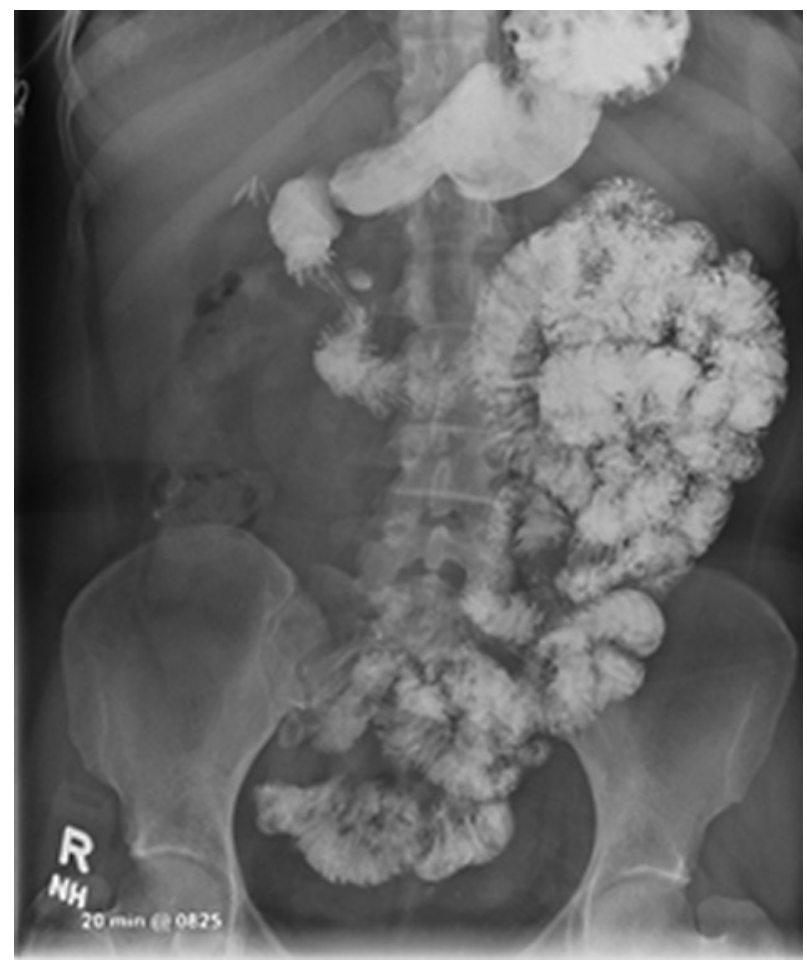

loperamide as high as $40 \mathrm{mg} 5$ times daily needed to control ileostomy output and prevent AKI $[7,8]$. Ileostomy creation leading to recurrent AKIs is often observed in the setting of short bowel syndrome. However, the timing of AKI in this patient following cryptosporidium infection and treatment makes ileostomy creation and short bowel syndrome less likely to be the main causes [9-11]. Additionally, BUN/Cr ratios $<20$ indicate that prerenal azotemia may not have been the primary cause of AKI. Ileostomy output remaining high in between hospitalizations further suggests that the underlying condition was likely not addressed until the final hospitalization.

Postinfectious symptoms from cryptosporidium are common even among healthy patients without IBD and who are not on immunosuppressants. In retroactive studies by both Hunter and Rehn, a significant portion of participants experienced both intestinal and nonintestinal symptoms in the months following infection with Cryptosporidium species. Recurrent gastrointestinal symptoms following acute cryptosporidium infection have been reported in up to $40 \%$ of immunocompetent participants [12]. These case patients' experience with one or more symptoms of vomiting, diarrhea, and abdominal pain within 2 months of initial infection supports the patient presentation in our case. The role of postinfectious inflammatory syndrome in the development of kidney injury may possibly be explained by the mechanism of direct cytopathic injury as observed in cases of pyogenic, leptospirosis, and nematode infections, among others [13]. The importance of detection and aggressive treatment in preventing renal injury as echoed in the contexts of various infectious agents and several renal clinical syndromes serves to further guide our understanding of the clinical course of this case patient [13].

The role of multimodal treatment then is highlighted by the final hospitalization. With multiple GI comorbidities present, extensive workup and treatments for multiple possible conditions were requisite. This case, therefore, demonstrates the importance underlying the exclusion of pathologies in complicated patients. If insurance had approved the octreotide 
earlier, perhaps the extent to which short bowel syndrome was involved could have been better understood and treatment ensued. Failure to obtain Crohn's workup in previous hospitalizations resulted in the overlooking of other possible etiologies. It was not until Crohn's exacerbation was proven unlikely that the diagnosis of postinfectious inflammatory syndrome was considered and, thus, treated successfully with the course of steroids. This emphasizes the need for consideration of a broad differential, especially in complicated patients, as demonstrated in this case.

\section{Statement of Ethics}

Written informed consent was obtained from the patient for publication of this case report and any accompanying images.

\section{Conflict of Interest Statement}

The authors have no conflicts of interest to declare.

\section{Funding Sources}

The authors have no funding sources to disclose.

\section{Author Contributions}

M.A. and A.K. wrote the manuscript, conducted literature review, and edited each other's work. V.K. provided feedback and guidance throughout the entire process while making edits on all versions of the manuscript. All authors read and approved the final manuscript.

\section{References}

1 Törnblom H, Holmvall P, Svenungsson B, Lindberg G. Gastrointestinal symptoms after infectious diarrhea: a five-year follow-up in a Swedish cohort of adults. Clin Gastroenterol Hepatol. 2007 Apr;5(4):461-4.

2 Banerjee D, Deb R, Dar L, Mirdha BR, Pati SK, Thareja S, et al. High frequency of parasitic and viral stool pathogens in patients with active ulcerative colitis: report from a tropical country. Scand J Gastroenterol. 2009;44(3):325-31.

3 Waters WR, Palmer MV, Ackermann MR, Harp JA. Accelerated inflammatory bowel disease of TCR-alpha-deficient mice persistently infected with Cryptosporidium parvum. J Parasitol. 1997;83(3):460-4.

4 Colussi O, Rouen A, Seksik P, Cosnes J, Beaugerie L, Sokol H. Acute cryptosporidiosis as a cause of sudden recurrence of digestive symptoms in patients with Crohn's disease. J Crohns Colitis. 2010;4(6):669-70.

5 Marcial MA, Madara JL. Cryptosporidium: cellular localization, structural analysis of absorptive cells parasite membrane-membrane interactions in guinea pigs, and suggestion of protozoan transport by $\mathrm{M}$ cells. Gastroenterology. 1986;90(3):583-94.

6 Manthey MW, Ross AB, Soergel KH. Cryptosporidiosis and inflammatory bowel disease (experience from the Milwaukee outbreak). Dig Dis Sci. 1997;42(8):1580-6.

7 Mackowski A, Chen H-K, Levitt M. Successful management of chronic high-output ileostomy with high dose loperamide. BMJ Case Rep. 2015;2015:bcr2015209411.

8 Stankiewicz M, Gordon J, Rivera J, Khoo A, Nessen A, Goodwin M. Clinical management of ileostomy highoutput stomas to prevent electrolyte disturbance, dehydration and acute kidney injury: a quality improvement activity. J Stomal Therapy Australia. 2019;39(1):8-10.

9 Baker ML, Williams RN, Nightingale JMD. Causes and management of a high-output stoma. Colorectal Dis. 2011;13(2):191-7. 
10 Parrish CR. The clinician's guide to short bowel syndrome. J Nurse Life Care Planning. 2016;16(2):11-38.

11 Ungvarsky J. Short bowel syndrome. Salem Press; 2019.

12 Hunter PR, Hughes S, Woodhouse S, Nicholas R, Syed Q, Chalmers RM, et al. Health sequelae of human cryptosporidiosis in immunocompetent patients. Clin Infect Dis. 2004;39(4):504-10.

13 Prasad N, Patel MR. Infection-induced kidney diseases. Front Med. 2018;5:327. 